\title{
PAPER
}

\section{Simultaneous estimation of source number and directions-of-arrival via blind beamforming}

\author{
Jie Zhuo* and Chao Sun \\ Institute of Acoustic Engineering, Northwestern Polytechnical University, \\ Xi'an, Shaanxi, 710072, P.R. China
}

(Received 13 May 2003, Accepted for publication 17 September 2003 )

\begin{abstract}
Blind beamforming algorithms have the ability to recover the desired signals from sensor array outputs without any prior knowledge of the direction-of-arrivals (DOAs). Non-Gaussian signals with negative kurtosis can be automatically captured by the multistage constant modulus (CM) array, which is the most striking blind beamforming algorithm and has been widely discussed in literatures. However, the sources number must be pre-determined in all kinds of blind beamforming algorithms. Based on the multistage CM array, we present a new method in this paper. It is designed to recover the desired signals and automatically determine the number of sources simultaneously. If the array geometry is known, the DOAs of all sources also can be estimated at the same time. The performance of the new method was analyzed via computer simulations and water tank experiments, and compared with that of other DOA estimation algorithms including "non-blind" and "blind" ones under the assumption of knowing the sources number. The new method shows better results in all considered situations.
\end{abstract}

Keywords: Blind beamforming, Multistage CM array, Signal detection, DOA, Water tank experiments

PACS number: 43.30.Wi, 43.60.Gk [DOI: 10.1250/ast.25.24]

\section{INTRODUCTION}

Directions-of-arrival (DOAs) estimation of multiple signals impinging on a sensor array is a well-studied problem in array signal processing. Traditional methods, such as MUSIC [1] and ESPRIT [2], exploit knowledge of the array geometry without using information of the signal itself. However, the assumption of a known array response is seldom satisfied in practice, which makes the DOA estimation performance of these traditional methods degrade significantly [3]. Recently, some methods are studied to recover the information of signals incident onto the array by exploiting their own properties only, such as high-order statistics [4], cyclostationarity [5], or constant modulus (CM) property [6]. Beamforming based on these methods are called blind beamforming, since no knowledge of the array geometry is required. After blind separation of the signals, the DOA estimation problem is decoupled and can be carried out for each source individually [7]. Blind beamforming methods are more robust to array manifold errors due to the extra information used. Since the pioneering work of Treichler and Agee [8], it is known

*e-mail: zpisces@hotmail.com that the CM property is a strong property that, by itself, is sufficient for source separation. Such a scheme is proposed in [9], where the CM signals are successively separated using the so-called multistage CM array. One stage of the multistage CM array consists of a conventional weightand-sum adaptive beamformer whose weights are updated by the constant modulus algorithm [8] without using any training signal. An adaptive signal canceller is included in each stage to remove a captured source from the input to next stage. In this way, several narrowband source signals can be sequentially extracted at successive stages of the system. If the array geometry is known, the DOAs can also be estimated [10]. Signal separation and reconstruction do not require knowledge of the array configuration.

The multistage CM array has fast convergence properties and low computational complexity. Moreover, the nonGaussian signals with negative kurtosis can be automatically captured by the CM array [11]. The determination of the number of source signals impinging on the array is a prerequisite to determine the order of the multistage $\mathrm{CM}$ array. In this paper, we present a new method based on the multistage CM array. It is designed to recover the signals and automatically determine the number of sources at the same time. With the new method, we do not need to pre- 


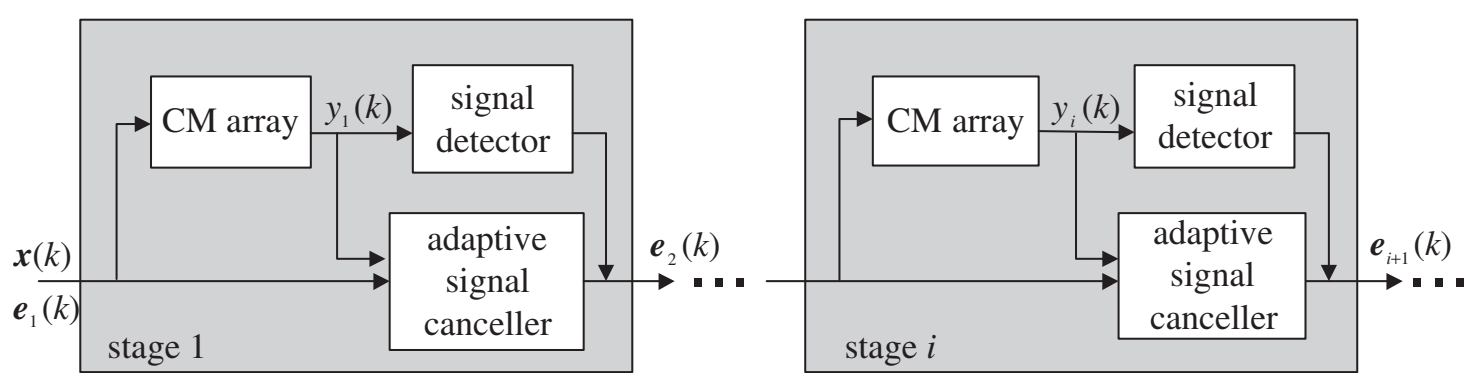

Fig. 1 Cascade multistage CM array with signal detector.

determine the order of the multistage CM array. Results of computer simulations and water tank experiments are presented to illustrate the effectiveness of the new method. We also compare the DOA estimation performance with other DOA estimation algorithms including "non-blind" and "blind" algorithms, which require to pre-determine the sources number.

This paper is organized as follows. Section 2 defines the data model. In section 3, we present the new method of the multistage $\mathrm{CM}$ array with signal detector. A DOAs estimation method is derived in section 4. Computer simulations and water tank experiments are presented in section 5, where the detection probability and the DOA estimation performance are illustrated and compared. Finally, concluding remarks are given in section 6 .

\section{DATA MODEL}

Consider a scenario in which several narrowband sources are contaminated by additive noise. Assume that there are $d$ non-Gaussian signals $\left\{s_{i}(k)\right\}_{i=1}^{d}$ with negative kurtosis impinging on an array of $M$ sensors from directions $\left\{\theta_{i}\right\}_{i=1}^{d}$. The $M$-sensors array may have arbitrary and unknown geometry and responses. Thus, the array input $\boldsymbol{x}(k)=\left[x_{1}(k), \ldots, x_{M}(k)\right]^{\mathrm{T}}$ can be written as

$$
\boldsymbol{x}(k)=\boldsymbol{a}\left(\theta_{1}\right) s_{1}(k)+\cdots+\boldsymbol{a}\left(\theta_{d}\right) s_{d}(k)+\boldsymbol{n}(k)
$$

or

$$
\boldsymbol{x}(k)=\boldsymbol{A s}(k)+\boldsymbol{n}(k)
$$

where superscript $\mathrm{T}$ denotes the transpose operation. $s(k)=$ $\left[s_{1}(k), \ldots, s_{d}(k)\right]^{\mathrm{T}}$ is the source signal vector, $\boldsymbol{n}(k)=$ $\left[n_{1}(k), \ldots, n_{M}(k)\right]^{\mathrm{T}}$ is an additive Gaussian noise vector with unknown variance. $\boldsymbol{s}(k)$ and $\boldsymbol{n}(k)$ are assumed to be temporally uncorrelated. $\boldsymbol{A}=\left[\boldsymbol{a}\left(\theta_{1}\right), \ldots, \boldsymbol{a}\left(\theta_{d}\right)\right]$ is referred to as the array manifold, which contains the information about the array geometry and responses and the directions of the impinging sources. Its column vector $\boldsymbol{a}\left(\theta_{i}\right)$ is the array response to the ith source, and called the direction vector.

\section{MULTISTAGE CM ARRAY AND SIGNAL DETECTOR}

A model of the cascade multistage $\mathrm{CM}$ array with signal detector is shown in Fig. 1. Each CM array output is represented by $y(k)$, which also is the recovered signal to one of incident signals. According to the constant modulus algorithm [8], the cost function of CM array can be written as:

$$
J(k)=E\left[|| y(k)|-1|^{2}\right] \rightarrow \min
$$

Using the adaptive steepest-descent method and replacing the gradient vectors with their instantaneous estimates, the iterative procedure of the $\mathrm{CM}$ array can be described by the following equations:

$$
\left\{\begin{array}{l}
y(k)=\boldsymbol{w}^{\mathrm{H}}(k) \boldsymbol{x}(k) \\
e_{\mathrm{c}}(k)=y(k) /|y(k)|-y(k) \\
\boldsymbol{w}(k+1)=\boldsymbol{w}(k)+\mu_{\mathrm{CMA}} \boldsymbol{x}(k) e_{\mathrm{c}}{ }^{*}(k)
\end{array}\right.
$$

where superscript $\mathrm{H}$ denotes complex conjugate transpose, * denotes the complex conjugate. Function (4) is a realization of the LMS algorithm with a desired signal $y(k) /|y(k)|$. The step size $\mu_{\mathrm{CMA}}>0$ controls the convergence rate of CM array.

The adaptive signal canceller can directly use the complex LMS algorithm, and be updated by

$$
\left\{\begin{array}{l}
y(k)=\boldsymbol{w}^{\mathrm{H}}(k) \boldsymbol{x}(k) \\
\boldsymbol{e}(k)=\boldsymbol{x}(k)-\boldsymbol{u}(k) y(k) \\
\boldsymbol{u}(k+1)=\boldsymbol{u}(k)+2 \mu_{\mathrm{LMS}} y^{*}(k) \boldsymbol{e}(k)
\end{array}\right.
$$

The step size is bounded by $0<\mu_{\mathrm{LMS}}<1 / \sigma_{y}{ }^{2}$, where $\sigma_{y}{ }^{2}=E\left[|y(k)|^{2}\right]$ is the variance of the CM array output at the current stage. Thus, the convergence properties of the canceller weights depend on weights of the CM array, whereas the $\mathrm{CM}$ array weights are independent of those of the adaptive canceller.

The determination of the number of sources impinging on the array is a prerequisite to determine the order of the multistage CM array stages. In this paper, a signal detector is included in each CM array to avoid such a procedure. The signal detector is used to evaluate the output level of each $\mathrm{CM}$ array and decide whether there is a signal 


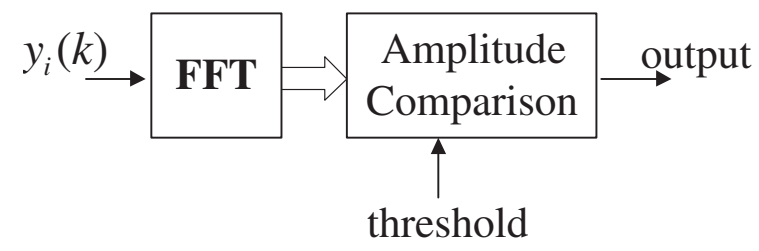

Fig. 2 Signal detector.

component present. Therefore the problem of detecting narrowband signals in additive noise becomes a spectral analysis procedure, which is usually implemented by the Fast Fourier Transform (FFT) [12].

The block diagram of the signal detector is shown in Fig. 2. After performing FFT to the CM array output signal $y(k)$, the strongest amplitude in the spectrum is compared to a threshold, which is obtained by setting a constant false alarm probability. If the threshold is exceeded, the detector indicates the presence of a narrowband signal and the signal separation will carry on; otherwise, the detector declares that no more sources exist and the procedure will stop. The number of incident sources is one less than the order of the multistage CM array.

\section{DOA ESTIMATION}

In some applications, estimation of the array manifold $\boldsymbol{A}$ is desirable. After blindly obtaining the array manifold $\hat{\boldsymbol{A}}$, we can estimate the DOA of each source by projecting each column vector of $\hat{\boldsymbol{a}}\left(\theta_{i}\right)$ of $\hat{\boldsymbol{A}}$ onto the array manifold. $\boldsymbol{A}$ can be obtained by computing the pseudo-inverse matrix of weight matrix $\boldsymbol{W}$ constructed in the blind beamforming procedure. In this paper, based on blind source reconstruction, the least-squares estimate of $\boldsymbol{A}$ is presented. Having the estimated signals $\hat{\boldsymbol{S}}=\left[\hat{\boldsymbol{s}}_{1}, \ldots, \hat{\boldsymbol{s}}_{d}\right]^{\mathrm{T}}$ at hand, $\boldsymbol{A}$ can be estimated by

$$
\hat{\boldsymbol{A}}=\arg \min _{A}\left\{(\boldsymbol{X}-\boldsymbol{A} \hat{\boldsymbol{S}})\left(\boldsymbol{X}-\boldsymbol{A} \hat{\boldsymbol{S}}^{\mathrm{H}}\right\}\right.
$$

Then we can obtain the optimum solution of $\boldsymbol{A}$

$$
\hat{\boldsymbol{A}}=X \hat{\boldsymbol{S}}^{\mathrm{H}}\left(\hat{\boldsymbol{S}} \hat{\boldsymbol{S}}^{\mathrm{H}}\right)^{-1}
$$

By the column vectors $\left\{\hat{\boldsymbol{a}}\left(\theta_{i}\right)\right\}_{i=1}^{d}$ of $\hat{\boldsymbol{A}}$, the DOAs are given by

$$
\theta_{i}=\arg \max _{\theta}\left|\hat{\boldsymbol{a}}\left(\theta_{i}\right)^{\mathrm{H}} \boldsymbol{a}(\theta)\right|^{2}, \quad i=1, \ldots, d
$$

where $\boldsymbol{a}(\theta)$ is the scanning vector, and $\theta$ varies within the searching space.

\section{EXPERIMENTS RESULTS}

Computer simulations and water tank experiments were conducted to investigate the detection and DOA estimation performance of the multistage $\mathrm{CM}$ array with the signal detector (abbreviated to CMA-SD). A uniform linear array (ULA) was assumed in simulation work and used in the experiments so as to be consistent with the theoretical work in earlier sections, and the results are presented in this section.

In all experiments, the $i$ th $\mathrm{CM}$ array weight was initialized to $\boldsymbol{w}_{i}(0)=\boldsymbol{i}$ to avoid the CM arrays converge to the same signal. The $i$ th element of the unit vector $i$ was 1 and all others were zero. The step size was set to $\mu_{\mathrm{CMA}}=0.005$. Signal canceller weights were initialized as $\boldsymbol{u}(0)=\boldsymbol{0}$ and the initial step size $\mu_{\mathrm{LMS}}=0.02$. The threshold was determined at a false alarm probability of signal detector of 0.01 .

\subsection{Computer Simulations}

In the computer simulations, a uniform linear array (ULA) of 16 identical isotropic sensors spaced a half wavelength apart was used. Assume that there are 3 independent sinusoids impinging on the array from directions $-10^{\circ}, 20^{\circ}$ and $10^{\circ}$. Additive noise is assumed as spatial white Gaussian noise with variance $\sigma_{n}{ }^{2}=1$. The number of snapshots used in simulations was 3,000.

The every stage beampattern and the convergence curve of CMA-SD are shown in Fig. 3, where the input SNR of each source was $5 \mathrm{~dB}$. The cost function of every stage $\mathrm{CM}$ array may be monitored using the following recursion:

$$
c_{i}(k+1)=\lambda c_{i}(k)+(1-\lambda)(|y(k)|-1)^{2}
$$

where $0<\lambda<1$ is the forgetting factor, and the update term corresponds to an instantaneous estimate of the original cost function (3). In the simulations, we set $\lambda=0.95$. Observing that convergence was quite fast, $\hat{s}_{1}(k)$ was captured after 500 samples. And the follow-on stages convergence was faster than the first one.

Changing the input SNR of three sources, Fig. 4 illustrates the detection probability and the DOA estimate bias of the source arrived from $10^{\circ} .100$ independent computer runs were performed at each input SNR. From the figure, the correct detection probability was higher than 0.7 when the input SNR was higher than $-15 \mathrm{~dB}$. Increasing the input SNR to $-10 \mathrm{~dB}$, the correct detection probability was approximate to 1 , and the bias of DOA estimate approached zero. Results show that the blind signal detection method based on the multistage CM array was very efficient. When the input SNR was higher than the SNR threshold, CMA-SD could correctly detect the signals and estimate their DOAs.

\subsection{Water Tank Experiments}

Water tank experiments were conducted in the anechoic water tank of Northwestern Polytechnical University, whose size is $20 \mathrm{~m} \times 8 \mathrm{~m} \times 7 \mathrm{~m}$. A 16-element ULA with $10 \mathrm{~cm}$ inter-element spacing was used as the receiving array. Two sources were placed at distances from the array. 


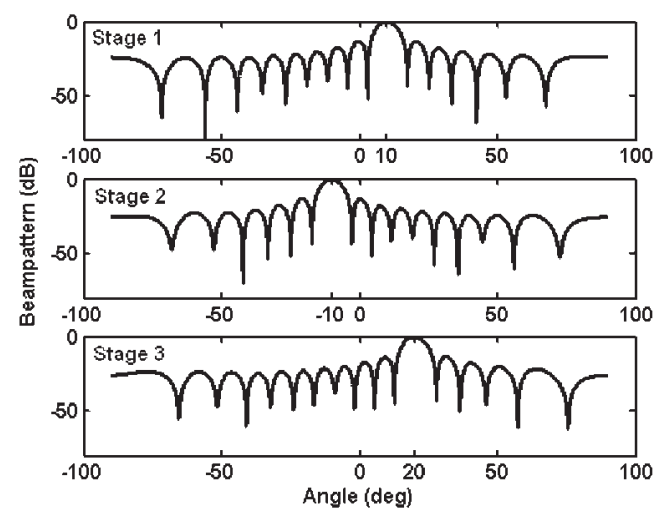

(a)

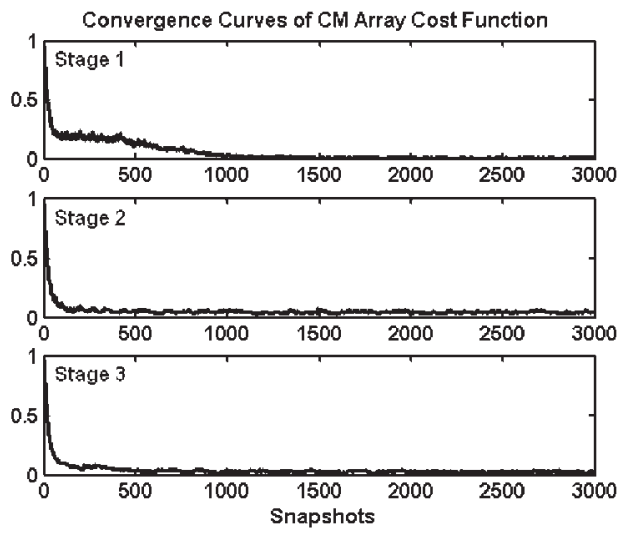

(b)

Fig. 3 16-sensors ULA receive three independent signals from direction $\left(-10^{\circ}, 20^{\circ}, 10^{\circ}\right)$. The inputs SNRs are all equal to $5 \mathrm{~dB}$. (a) Beampatterns of CM array, and (b) Convergence curves of CM array cost function.

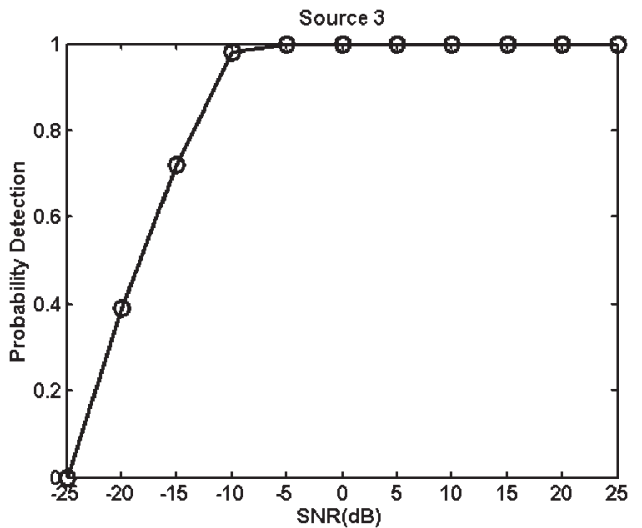

(a)

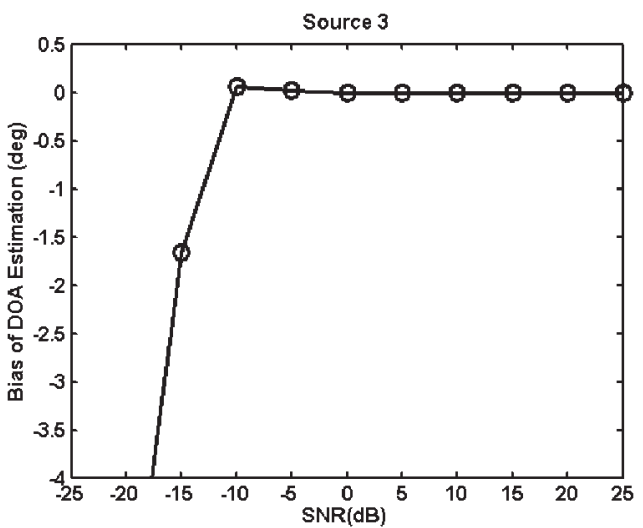

(b)

Fig. 4 Change the input SNR of three sources from $-25 \mathrm{~dB}$ to $25 \mathrm{~dB}$, the performance curves of the source arrived from $10^{\circ}$. (a) Curve of detection probability versus SNR; (b) Curve of the DOA estimation bias.

During the experiments, the position of one source was fixed while the other one was placed at different positions to generate signals from varying directions. The sample frequency was $51.2 \mathrm{kHz}$ through the experiments. Because the experiments were designed to examine the CMA-SD performance for two independent sources, two sources were excited by two different sinusoids at frequencies of $10 \mathrm{kHz}$ and $9 \mathrm{kHz}$ to simulate two uncorrelated sources.

In the experiments, DOA estimation performance of four algorithms including conventional beamforming (abbreviated to CBF), MUSIC, cumulant-based blind beamforming (abbreviated to CUM), and the CMA-SD was compared. The former two non-blind algorithms estimate DOAs based on the spatial spectrum, and the other two blind beamforming algorithms do it by projecting each direction vector $\hat{\boldsymbol{a}}\left(\theta_{i}\right)$ onto the array manifold. Except the CMA-SD, the other algorithms assumed the sources number. In off-line data processing, data from total 16 sensors and those only from the central 8 sensors were used respectively, to compare the performance of four algo-

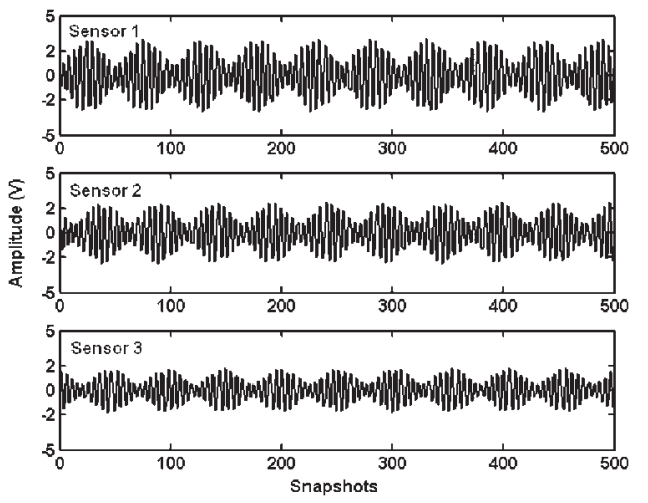

Fig. 5 Output sample signal amplitude at the first, second and third sensor.

rithms in different situations. The results presented were average over 100 independent runs, with a sample data length of 3,072 in each run.

Fixing two sources in $-22.5^{\circ}$ and $-9.1^{\circ}$, Fig. 5 shows the signal amplitudes at the first three sensors. Inconsis- 
tency in the gains is evident. Other errors including sensor position errors and phase errors were also present. To compare the robustness of different algorithms, no array calibration and corresponding modification was conducted before data processing.

The spectrum of outputs at the third sensor is illustrated in Fig. 6. Two sources were not of equal-power. Firstly, data from total 16 sensors were analyzed. Figures 7(a) and (b) show the beampatterns at each stage of CMA-SD. Figures 8(a) and (b) present the convergence curves of the cost functions at each stage of CMA-SD and the spectra of the reconstructed signals. The detection probability of CMA-SD for two sources is presented in Table 1, and the DOA estimate means obtained from different algorithms are also given in Table 1, based on 100 independent experiment data records at each sources direction. Secondly, results using the data from the central 8 sensors are shown in Table 2. Two sources were not of equal-power, so the detection probability of the source with lager power

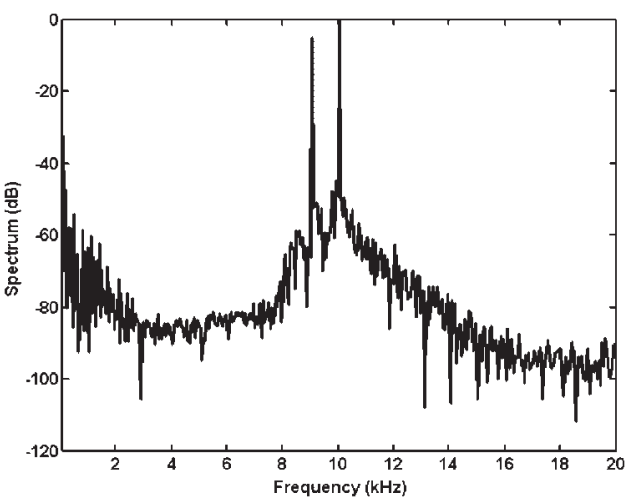

Fig. 6 The output sample signal spectrum at the third sensor.

was equal to 1 , higher than that of the weaker source. Detection probability of the weaker source was decreased when reducing the separation between the sources.

Decreasing the number of sensors is equivalent to increasing the Rayleigh beamwidth of the receive array. At

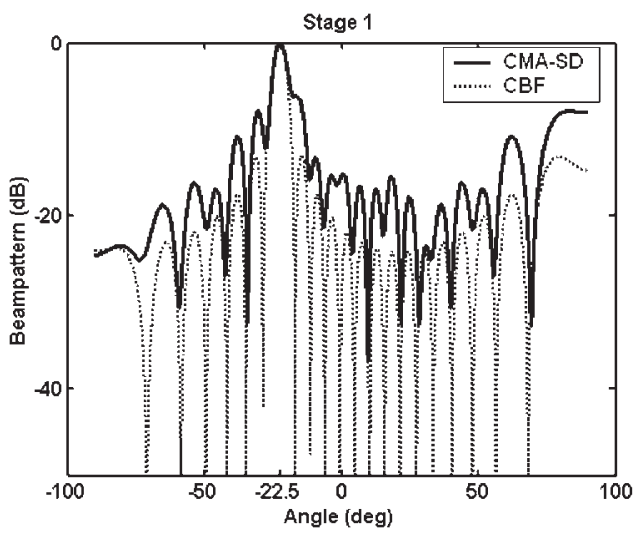

(a)

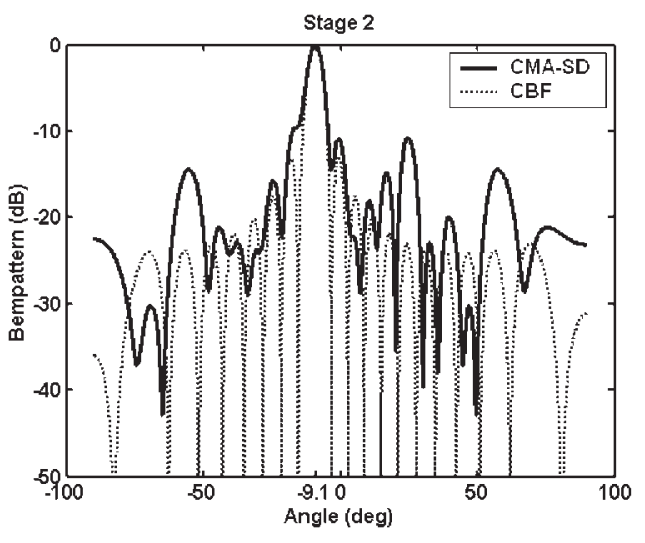

(b)

Fig. 7 Beampattern of each $\mathrm{CM}$ array. The sensors number is 16, and two independent signals from directions $\left(-22.5^{\circ},-9.1^{\circ}\right)$. (a) the first $\mathrm{CM}$ array, and (b) the second $\mathrm{CM}$ array.
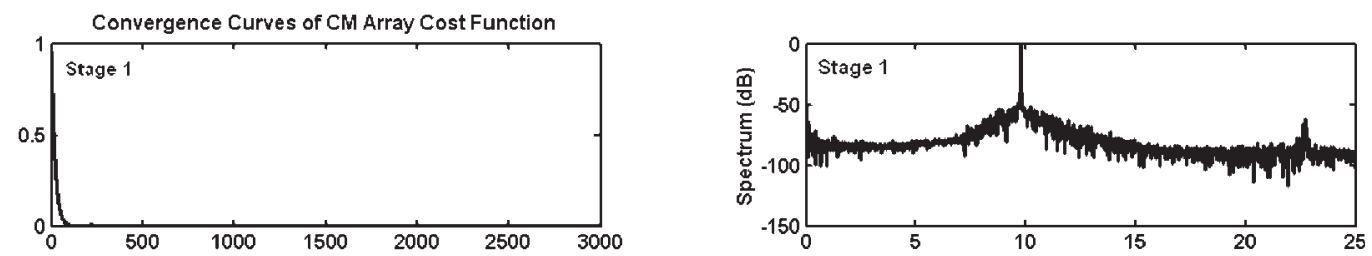

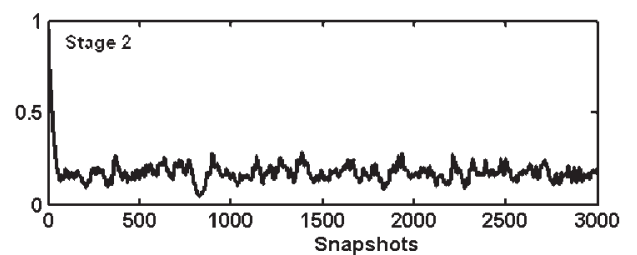

(a)

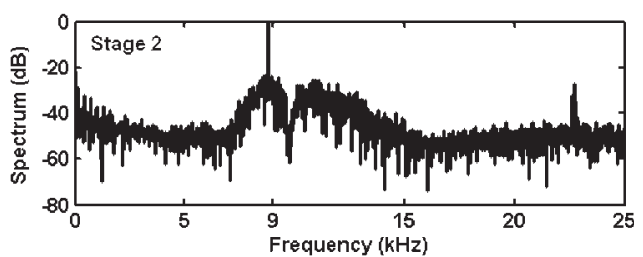

(b)

Fig. 8 16-sensors ULA receive two independent signals from directions $\left(-22.5^{\circ},-9.1^{\circ}\right)$. (a) Convergence curve of each CM array cost function, and (b) Spectra of CM array outputs. 
Table 1 DOA estimation means of different algorithms with the 16 sensors $\left({ }^{\circ}\right)$.

\begin{tabular}{cccc}
\hline & $(-22.5,-9.1)$ & $(-22.5,-15.0)$ & $(-22.5,-17.8)$ \\
\hline $\begin{array}{c}\text { Detection } \\
\text { probability }\end{array}$ & $(1,1)$ & $(1,0.96)$ & $(1,0.97)$ \\
CMA-SD & $(-22.40,-9.10)$ & $(-22.49,-15.07)$ & $(-22.50,-18.92)$ \\
CBF & $(-22.40,-9.30)$ & $(-22.50,-16.00)$ & $(-22.60,-18.70)$ \\
MUSIC & $(-22.40,-9.30)$ & $(-22.40,-15.40)$ & $(-22.20,-19.40)$ \\
CUM & $(-22.50,-9.20)$ & $(-22.50,-15.10)$ & $(-22.60,-19.00)$ \\
\hline
\end{tabular}

Table 2 DOA estimation means of different algorithms with the central 8 sensors $\left({ }^{\circ}\right)$.

\begin{tabular}{cccc}
\hline & $(-22.5,-9.1)$ & $(-22.5,-15.0)$ & $(-22.5,-17.8)$ \\
\hline Detection & $(1,1)$ & $(1,0.94)$ & $(1,0.92)$ \\
probability & $(-22.40,-9.71)$ & $(-22.47,-14.00)$ & $(-22.50,-17.92)$ \\
CMA-SD & $(-22.50,-9.20)$ & $(-22.00,18.00)$ & $(-21.40,17.30)$ \\
CBF & $(-22.20,-9.60)$ & $(-21.90,-14.16)$ & $(-21.80,17.40)$ \\
MUSIC & $(-22.20)$ \\
CUM & $(-22.34,-9.70)$ & $(-22.50,-14.20)$ & $(-22.60,-18.40)$ \\
\hline
\end{tabular}

frequency of $10 \mathrm{kHz}$, the Rayleigh beamwidth of 16 sensors ULA is $4.5^{\circ}$ and one of 8 -sensors ULA is $9.1^{\circ}$. Due to decreasing the number of sensors and system errors in the receive array, CBF and MUSIC were ineffective at small source separations. On the other hand, the blind algorithms perform well in all situations. And also, the DOA estimation errors of CMA-SD are the smallest among four algorithms under consideration. The water tank experiments results show that CMA-SD can correctly detect signals and estimate the DOAs at the same time when signals are recovered.

\section{CONCLUSIONS}

In this paper, we present a new method based on the cascade multistage CM array to automatically detect the number of sources and estimate their DOAs at the same time when signals are recovered. A signal detector is added behind each CM array to decide whether there is a signal present in the output, therefore the multistage CM array need not pre-determine the order of the system. We analyzed the detection probability of the multistage CM array with the signal detector by means of computer simulations and water tank experiments, and also compared the DOA estimation performance of the new method with that of three other algorithms including "non-blind" and "blind" algorithms. Results show that the new method could recover the source signals and detect the number of sources at the same time with better performance.

\section{ACKNOWLEDGMENT}

This work is supported by the Doctorate Creation Foundation of Northwestern Polytechnical University (No. 200203).

\section{REFERENCES}

[1] R. Schmidt, "A signal subspace approach to multiple emitter location and spectral estimation," Ph.D. Dissertation, Stanford Univ. (1981).

[2] R. Roy and T. Kailath, "ESPRIT-Estimation of signal parameters via rotational invariance techniques," IEEE Trans. Acoust. Speech Signal Process., 38, 984-995 (1989).

[3] A. L. Swindlehurst and T. Kailath, "A performance analysis of subspace-based methods in the presence of model errors, Part I: The MUSIC algorithm," IEEE Trans. Signal Process., 40, 1758-1774 (1992).

[4] E. Gonen and J. M. Mendel, "Application of cumulants to array processing-Part III: Blind beamforming for coherent signals," IEEE Trans. Signal Process., 45, 2252-2264 (1997).

[5] L. Castedo and A. R. Figueiras-Vidal, "An adaptive beamforming technique based on cyclostationary signal properties," IEEE Trans. Signal Process., 43, 1637-1650 (1995).

[6] R. P. Gooch and J. D. Lundell, "The CM array: An adaptive beamformer for constant modulus signals," Proc. ICASSP, Tokyo, Japan, pp. 2523-2526 (1986).

[7] A. S. Kayhan and M. G. Amin, "Spatial evolutionary spectrum for DOA estimation and blind signal separation," IEEE Trans. Signal Process., 48, 791-798 (2000).

[8] J. R. Treichler and B. G. Agee, "A new approach to multipath correction of constant modulus signals," IEEE Trans. Acoust. Speech Signal Process., 31, 459-472 (1983).

[9] J. J. Shynk and R. P. Gooch, "The constant modulus array for co-channel signal copy and direction finding," IEEE Trans. Signal Process., 44, 652-660 (1996).

[10] A. Leshem and A. J. van der Veen, "Direction-of-arrival estimation for constant modulus signals," IEEE Trans. Signal Process., 47, 3125-3129 (1999).

[11] J. D. Lundell and B. Widrow, "Application of the constant modulus adaptive beamformer to constant and nonconstant modulus signals," Proc. 21st Asilomar Conf. Signals, Syst., Comput., Pacific Grove, CA, USA, pp. 432-436 (1987).

[12] Y. T. Chan, Q. Ma, H. C. So and R. Inkol, "Evaluation of various FFT methods for single tone detection and frequency estimation," IEEE 1997 Canadian Conf. Electrical and Computer Engineering, pp. 211-214 (1997). 